\title{
Proposição de valores de referência para a concentração natural de metais pesados em solos brasileiros
}

\author{
Francisco de S. Fadigas ${ }^{1}$, Nelson M. B. do Amaral Sobrinho ${ }^{2}$, Nelson Mazur ${ }^{2}$, Lúcia H. C. dos Anjos ${ }^{2} \&$ Alessandra A. Freixo ${ }^{3}$
}

\section{RESUMO}

Para avaliar a extensão da poluição de uma área, é comum se comparar os teores totais de metais pesados encontrados num dado solo com aqueles defrontados em condições naturais (não poluídos) ou com valores de referência (padrões). Propuseram-se através deste estudo: 1) valores de referência (VR) e limites de tolerância (LT) para os mais representativos solos brasileiros, e 2) um modelo para obtenção das concentrações naturais de $\mathrm{Cd}, \mathrm{Co}, \mathrm{Cr}, \mathrm{Cu}, \mathrm{Ni}, \mathrm{Pb}$ e $\mathrm{Zn}$ em um solo, a partir dos teores de silte, argila, Mn, Fe e CTC. Reuniram-se 256 amostras de solo por similaridade, em sete grupos e se calcularam as concentrações correspondentes ao quartil superior dos dados. Essas concentrações são propostas como valores de referência (VR) em solos brasileiros; além disso, obtiveram-se os limites de tolerância (LT) para cada grupo a partir da expressão antilog $(m+2 s)$, cujos $m$ (média) e $s$ (desvio padrão) dos dados foram transformados em $\log _{10}$. As funções de classificação da análise discriminante mostraram se apropriadas para alocar novas amostras nos grupos estabelecidos. Assim, é possível avaliar solos sob atividade antrópica e saber se há motivo para suspeitar de que a área esteja poluída.

Palvras-chave: poluição do solo, teores naturais, níveis de metais, metais em solos

\section{Proposition of reference values for natural concentration of heavy metals in Brazilian soils}

\begin{abstract}
To evaluate the extension of soil pollution of a site it is common to compare the contents found in a specific soil with those in natural conditions (unpolluted) or with reference values (patterns). The goal of this study was to propose: 1) reference values (VR) and tolerance limits for the most representative Brazilian soils, and 2) a model for obtaining the levels of $\mathrm{Cd}$, $\mathrm{Co}, \mathrm{Cr}, \mathrm{Cu}, \mathrm{Ni}, \mathrm{Pb}$ and $\mathrm{Zn}$ in a soil under natural conditions from the silt, clay, $\mathrm{Mn}$, Fe contents and $\mathrm{CEC}$ value. A set of 256 soil samples was formed by similarity in seven groups and the concentrations corresponding to the upper quartile of the data were calculated. These concentrations are proposed as the reference values (VR) in Brazilian soils. Besides, tolerance limits admitted for each group were taken as antilog $(m+2 s)$, where $m$ (average) and $s$ (standard deviation) were transformed to $\log _{10}$. The classification functions of the discriminant analysis proved to be appropriate to allocate new samples in the established groups. Thus, it is possible to evaluate soils under anthropogenic activity and to know if there is a reason for suspecting of pollution of the site.
\end{abstract}

Key words: soil pollution, baselines levels, metal levels, metals in soil

1 Departamento de Química Agrícola e Solos - UFBA, CEP.44 380-000, Cruz das Almas, BA. Fone: (75) 26211220. E-mail: fadigas@ufba.br 2 Departamento de Solos - UFRRJ -, BR 465, Km 7, CEP.23850 000, Seropédica, RJ. Fone (21) 37873755. E-mail: nelmoura@ufrrj.br :

${ }^{3}$ Departamento de Botânica - UEFS, Av. Universitária, s/n - Km 03 da BR 116, CEP: 44031-460 Feira de Santana, BA. Fone (75) 2248200. E-mail: freixo@uefs.br 


\section{INTRODUÇÃO}

Para se avaliar a extensão da poluição de uma área, é comum comparar os teores totais de metais pesados encontrados em um solo com aqueles defrontados em condições naturais (não poluídos) ou com valores de referência (padrões); todavia, no Brasil ainda não foi elaborado um sistema contendo padrões de referência de metais pesados no solo, para avaliação de áreas em relação à contaminação. Diferentes metodologias têm sido utilizadas para se estabelecer os valores de referência (VR) de metais pesados em solos, em que as mais comuns, são: o uso de valores considerados normais, citados na literatura; o estabelecimento de faixas de referência obtidas a partir de amostras controle, tomadas em áreas sem atividade antrópica, o mais longe possível de fontes de poluição (Casarini, 2000; Crock et al., 1992) e pela separação entre valores normais e anômalos, obtidos de uma coleção de dados que inclui solos contaminados e não contaminados (Tobias et al., 1997a; Wang, 1994).

O estabelecimento de faixas de concentração de referência e de limites máximos considerados normais no solo, tem sido feito por meio de diferentes procedimentos estatísticos (Fadigas et al., 2002). A expressão $L-$ antilog ( $m \pm 2 s)$, em que $m$ é a média e s é o desvio padrão dos dados transformados em $\log _{10}$, foi utilizada para calcular os limites superior e inferior dos valores de referência em solos do Alaska (Crock et al., 1992) e por Tobias et al. (1997a) para estimar faixas de concentração normal de alguns elementos traço no nordeste da Espanha. Valores de referência para o estado de São Paulo foram definidos com base no quartil superior (75\%) dos resultados analíticos (Lemos, 2000) e em solos da China, as concentrações de metais pesados em argilito foram usadas como padrões gerais (Wang, 1994). Técnicas da estatística multivariada como análise de agrupamento e análise discriminante foram utilizadas em estudos geoquímicos (Moura, 1985; Oliveira et al., 1998) e de inter relações entre atributos do solo e produtividade de cultivos (Correia, 1993), as quais apresentam potencial para uso no estabelecimento e proposição de valores de referência de metais em solos.

Alguns atributos do solo se destacam por sua relação com o conteúdo de metais pesados. Diversos autores encontraram correlações positivas entre o teor de argila e ferro e os teores totais de metais (Pérez et al., 1997; Rovers et al., 1983; Santos Filho \& Rocha, 1982; Souza et al., 1996; Valadares \& Catani, 1975; Valadares, 1975). Também pode haver associação entre metais pesados e a fração silte, visto que nesta fração do solo podem ser encontradas partículas finas (argila) e óxidos, devido à ineficiência no processo de dispersão dos solos durante a análise granulométrica (Klamt \& van Reeuwijk, 2000; Lima, 1984); por outro lado, nem sempre tem sido obtida correlação significativa com a matéria orgânica e o pH do solo (Furlani et al., 1977; Lavado et al., 1998; Qian et al., 1996).

Objetivou-se com este estudo, propor: 1) valores de referência (VR) e limites de tolerância (LT) para os mais representativos solos brasileiros e 2) um modelo para obtenção dos teores naturais de $\mathrm{Cd}, \mathrm{Co}, \mathrm{Cr}, \mathrm{Cu}, \mathrm{Ni}, \mathrm{Pb}$ e $\mathrm{Zn}$ em um solo, a partir dos teores de silte, argila, Mn, Fe e a CTC.

\section{MATERIAL E MÉTODOS}

Para este estudo, utilizou-se um conjunto de 256 amostras de solo, correspondendo aos horizontes A e B (ou C) de 110 perfis, das principais classes de solos encontradas no Brasil, especialmente os Argissolos e Latossolos (Tabela 1). Os solos selecionados estavam sob mínima ou nenhuma atividade antrópica. As análises de metais pesados foram feitas por extração com água régia e as determinações em Plasma de Acoplamento Indutivo (ICP-AES).

Tabela 1. Origem dos dados e tipo de extração utilizada por cada autor para obtenção das concentrações de alguns metais pesados em amostras de solos

\begin{tabular}{|c|c|c|c|}
\hline Autor & Camada & $\begin{array}{c}\mathrm{N}^{0} \text { de } \\
\text { Amostras }\end{array}$ & $\begin{array}{c}\text { Método de Extração } \\
\text { Extrator }\end{array}$ \\
\hline $\begin{array}{l}\text { Coleção de solos da } \\
\text { EMBRAPA }\end{array}$ & $\begin{array}{c}A \\
A / B, B / A \text { ou } E \\
B\end{array}$ & $\begin{array}{l}83 \\
30 \\
82\end{array}$ & Água régia (3:1) \\
\hline Pérez et al. (1997) & $\begin{array}{l}\text { A } \\
\text { B }\end{array}$ & $\begin{array}{l}4 \\
4\end{array}$ & Água régia (3:1) \\
\hline Oliveira (1996) & $\begin{array}{r}0-10 \mathrm{~cm} \\
50-60 \mathrm{~cm}\end{array}$ & $\begin{array}{l}20 \\
20\end{array}$ & Nitroperclórica (4:1) \\
\hline Ramalho et al. (1999) & $\begin{array}{r}0-10 \mathrm{~cm} \\
10-20 \mathrm{~cm}\end{array}$ & $\begin{array}{l}2 \\
2\end{array}$ & Água régia (3:2) \\
\hline Ramalho et al. (2000) & $\begin{array}{r}0-10 \mathrm{~cm} \\
10-20 \mathrm{~cm} \\
20-30 \mathrm{~cm}\end{array}$ & $\begin{array}{l}3 \\
3 \\
3\end{array}$ & Água régia (3:2) \\
\hline Total & & 256 & \\
\hline
\end{tabular}

Os resultados obtidos (médias das repetições) foram submetidos à análise de agrupamento (AC), utilizando-se os teores de silte, argila, Mn e Fe (pseudo-total) e CTC como variáveis, considerando-se que estas possuem boa relação com o teor dos metais em estudo (Fadigas, 2002). Estas variáveis apresentam distribuição assimétrica e foram transformadas em $\log _{10}$, embora a ausência de distribuição normal não seja considerada crítica em análise multivariada, quando se trabalha com grande número de amostras ou repetições (Statsoft, 1999). Para a análise de agrupamento, os dados transformados foram padronizados (valor observado menos a média/desvio padrão), para evitar a interferência das diferentes unidades de medida nos cálculos (Broschat,1979; Parks, 1966).

A escolha das variáveis foi baseada no estudo de correlações simples (Pearson) e no estudo de componentes principais, segundo Fadigas (2002). A análise foi processada em modo Q, ou seja, com o uso de um coeficiente de distância, para medir a similaridade entre as amostras, após a padronização dos dados. Para o agrupamento adotou-se a distância Euclidiana (distância geométrica entre dois objetos no espaço multidimensional) como medida de dissimilaridade. Embora o uso da distância Euclidiana possa causar distorção na estimativa da distância entre objetos quando são consideradas muitas variáveis (Cruz \& Regazzi, 2001), sua escolha neste estudo se justifica pela impossibilidade de se utilizar outras medidas, como a distância Euclidiana média 
ou à distância de Mahalanobis para um conjunto de 256 amostras nos softwares em que foram realizadas as análises estatísticas (SAEG 5.0, Statistica versão 5.5 e SPSS 10.0). Os casos foram ligados entre si pelo método de Ward (Statsoft, 1999), no qual se procura diminuir a soma de quadrados (SQ) dos grupos formados em cada etapa e é considerado muito eficiente.

Após o estabelecimento dos grupos utilizou-se a análise discriminante (AD) com o objetivo de avaliar o grau de acerto na alocação das amostras nos sete grupos e obter as funções de classificação que possibilitassem o enquadramento de novas amostras. Na análise discriminante, os dados não foram padronizados, pois nela a diferença entre unidades de medida não interfere nos resultados. Para a classificação das amostras empregou-se a distância generalizada de Mahalanobis (Statsoft, 1999) e a probabilidade proporcional ao tamanho do grupo. As análises foram processadas através do programa Statistica versão 5.5 (Statsoft, 1999). Os limites para os teores metais (Cd, $\mathrm{Co}, \mathrm{Cr}, \mathrm{Cu}, \mathrm{Ni}, \mathrm{Pb}$ ) considerados normais no solo em cada grupo, foram calculados adotando se o quartil superior (QS), ou seja, o valor correspondente a 75\% da distribuição da freqüência acumulada (Casarini, 2000) e os limites máximos obtidos pela expressão LT antilog $(m+2 s)$, em que LT - limite de tolerância, $m$ - média e $\mathrm{s}$ - desvio padrão dos valores em $\log _{10}$, respectivamente (Tobias et al., 1997a, 1997b).

\section{RESULTADOS E DISCUSSÃO}

\section{Obtenção de grupos de amostras por semelhança}

O estabelecimento de VR a partir de resultados analíticos de concentração de metais pesados, seja com base na média, intervalos de confiança ou quartil superior tem, como principal limitação, a diversidade dos solos encontrados no Brasil; assim, sua definição a partir das médias ou intervalos globais de concentração, não possuiria maior significado, uma vez que existe grande variabilidade desses teores entre os diferentes tipos de solo e, por conseqüência, seria obtida uma ampla faixa de variação. Da mesma forma o estabelecimento de um único limite máximo para concentrações normais baseado no quartil superior, não seria adequado para avaliação de solos cujas concentrações de metais fossem naturalmente baixas, indicando a necessidade de se utilizar uma metodologia que reúna solos com características semelhantes, especialmente aqueles atributos correlacionados com o teor de metais pesados. Para contornar este problema, as amostras de solo foram classificadas com base no seu grau de semelhança, em grupos homogêneos, levando se em conta a contribuição conjunta das variáveis silte, argila, Mn, Fe e CTC (Figura 1).

A interpretação do dendrograma considerando se um valor de 20 para o coeficiente de distância (Figura 1), permitiu que se identificasse sete grupos (G1 a G7) de amostras mais próximas entre si. As delimitações foram estabelecidas com base na análise visual do dendrograma, na qual se avaliaram os pontos de alta mudança de nível (Cruz \&

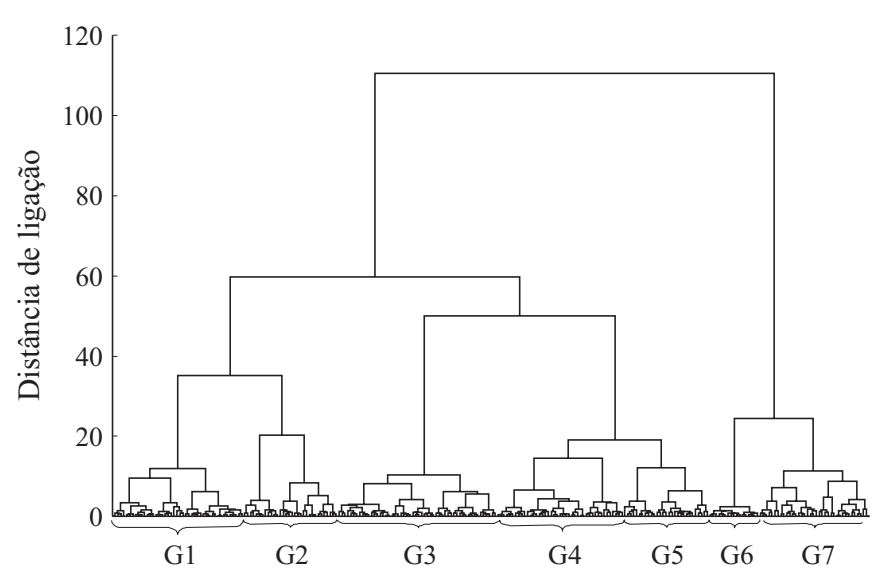

Figura 1. Dendrograma de classificação das amostras de solo em sete grupos em função dos teores de silte, argila, T, Fe e Mn

Regazzi, 2001; Moura, 1985). Analisando-se a composição dos grupos, constatou-se que todos se compunham tanto por amostras do horizonte $\mathrm{A}$ quanto de camadas intermediárias (A/B, B/A ou E) ou do B, em proporções variadas, indicando a coerência de se utilizar os atributos do solo que têm relação com o teor de metais pesados na formação dos grupos, sem considerar a qual horizonte do solo cada amostra pertence.

Os grupos obtidos por semelhança entre as amostras também se compõem de solos de diferentes classes pedológicas, uma vez que solos de classes pedológicas diferentes podem apresentar alguns atributos químicos e de composição granulométrica em comum (EMBRAPA, 1999); assim, amostras de horizontes A, B ou transicionais de um mesmo solo ou de solos distintos, podem estar presentes em um mesmo grupo, desde que as concentrações de algumas das variáveis consideradas na análise de agrupamento, sejam próximas (Tabela 2). Alguns autores observaram que o teor de metais também varia entre solos pertencentes a uma mesma classe, ou entre solos de classes diferentes, em função das variações nas características dos solos (Amaral Sobrinho, 1993; Mazur, 1997; Ramalho et al., 2000; Valadares, 1975).

A análise das médias obtidas para as variáveis constantes na Tabela 2 permite que as variáveis que melhor caracterizam cada grupo, sejam identificadas, deste modo, pode se dizer que o grupo 1 (G1) é caracterizado pelas maiores concentrações de Mn e Fe, seguidos por um teor de argila próximo daqueles encontrados nos grupos 2 (G2) e 3 (G3). Este grupo (G1), é composto, predominantemente, por amostras de Latossolo Vermelho Distroférrico, Nitossolo Vermelho Distroférrico, Latossolo Bruno e Argissolo Vermelho Eutroférrico, formados sobre basalto ou materiais derivados (Oliveira, 1996), naturalmente ricos em metais pesados (Alloway, 1995; Ross, 1994; Oliveira et al., 1998; Valadares, 1975) fazem parte deste grupo, ainda, amostras de Latossolo Vermelho Distrófico, Argissolo Vermelho, Luvissolo e Cambissolo, originados de basalto, migmatito, biotita gnaisse e argilito da Formação Solimões. Os teores mais elevados de $\mathrm{Mn}$ e Fe neste grupo refletem a origem dos solos que os compõem. 
Tabela 2. Caracterização dos grupos formados pelas amostras de solo em função dos seus teores de silte, argila, ferro e manganês e da CTC (a pH 7,0)

\begin{tabular}{|c|c|c|c|c|c|c|c|c|c|c|c|}
\hline \multirow{4}{*}{$\begin{array}{l}\text { Grupo } \\
\text { (G) }\end{array}$} & \multirow{4}{*}{$\begin{array}{c}\mathrm{N}^{0} . \\
\text { Casos }^{1}\end{array}$} & \multicolumn{10}{|c|}{ Variáveis de solo } \\
\hline & & \multirow{2}{*}{\multicolumn{2}{|c|}{$\frac{\text { CTC }}{\mathrm{cmol}_{\mathrm{c}} \mathrm{kg}^{-1}}$}} & \multicolumn{2}{|c|}{ Silte } & \multirow{2}{*}{\multicolumn{2}{|c|}{$\begin{array}{l}\text { Argila } \\
\mathrm{g} \mathrm{kg}^{-1}\end{array}$}} & \multirow{2}{*}{\multicolumn{2}{|c|}{$\mathrm{Fe}$}} & \multirow{2}{*}{\multicolumn{2}{|c|}{$\frac{\mathrm{Mn}}{\mathrm{mg} \mathrm{kg}^{-1}}$}} \\
\hline & & & & & & & & & & & \\
\hline & & $\mathrm{m}^{2}$ & $\mathrm{~s}^{3}$ & m & $s$ & $\mathbf{m}$ & s & $\mathbf{m}$ & s & $\mathbf{m}$ & $s$ \\
\hline 1 & 44 & 8,8 & $\pm 4,4$ & 195 & \pm 63 & 456 & \pm 128 & 72,7 & $\pm 34,4$ & 522,3 & $\pm 213,4$ \\
\hline 2 & 32 & 17,1 & $\pm 6,1$ & 264 & \pm 120 & 468 & \pm 208 & 20,9 & $\pm 6,4$ & 305,2 & $\pm 283,2$ \\
\hline 3 & 54 & 5,7 & $\pm 2,1$ & 98 & \pm 35 & 575 & \pm 173 & 40,2 & $\pm 15,3$ & 93,7 & $\pm 64,6$ \\
\hline 4 & 43 & 7,0 & $\pm 2,5$ & 157 & \pm 70 & 259 & \pm 94 & 22,3 & $\pm 7,9$ & 219,0 & $\pm 174,1$ \\
\hline 5 & 29 & 5,3 & $\pm 2,2$ & 132 & \pm 47 & 154 & \pm 81 & 9,2 & $\pm 4,2$ & 76,4 & $\pm 36,8$ \\
\hline 6 & 17 & 2,7 & $\pm 0,5$ & 74 & \pm 13 & 305 & \pm 44 & 14,6 & $\pm 3,8$ & 40,7 & $\pm 10,3$ \\
\hline 7 & 37 & 4,5 & $\pm 2,2$ & 47 & \pm 24 & 139 & \pm 68 & 6,5 & $\pm 3,8$ & 43,6 & $\pm 35,7$ \\
\hline
\end{tabular}

${ }^{1}$ Número de amostras consideradas nos cálculos, ${ }^{2}$ Média aritmética, ${ }^{3}$ Desvio padrão

O grupo 2 se diferencia dos demais, especialmente por seus teores de silte e Mn e pela CTC. Deste grupo participam diversos solos com CTC elevada, como Chernossolos, Luvissolos e solos eutróficos, como Planossolo Háplico Eutrófico, Plintossolo Argilúvico Eutrófico e Gleissolo Háplico Eutrófico; ressalta-se ser ele composto, ainda, por amostras de Latossolo Amarelo, Latossolo Vermelho Amarelo e Argissolo Vermelho.

Outro grupo que se destaca é o grupo 7 (G7) no qual são encontradas as menores concentrações médias das variáveis utilizadas para o agrupamento das amostras de solos, exceto a CTC. Em termos gerais, os grupos G6 e G7 são similares em composição, mas se diferenciam pelas concentrações de Argila e Fe; neles se encontram principalmente amostras de Latossolos e Argissolos Amarelos Distróficos, seguidas por pequeno número de Latossolos e Argissolos Vermelho Amarelos Distróficos e Planossolo Háplico Distrófico, originados predominantemente de sedimentos Terciários e Quaternários.

Os grupos G3, G4 e G5 apresentam características intermediárias entre G1 e G6/G7 e são constituídos por amostras de grande variedade de Latossolos e Argissolos e, em menor quantidade, por amostras de Plintossolo, Cambissolo e Nitossolo, com predominância acentuada do caráter distrófico.

O uso da análise de agrupamento possibilitou a separação de grupos mais ou menos uniformes e indica que, com base nas características dos solos presentes em cada grupo (silte, argila, CTC, Mn e Fe) e que possuem relação com a concentração dos metais pesados no solo, será possível obter-se as concentrações naturais de $\mathrm{Cd}, \mathrm{Co}, \mathrm{Cr}, \mathrm{Cu}, \mathrm{Ni}, \mathrm{Pb}$ e Zn, para uma ampla variedade de solos brasileiros.

\section{Valores propostos como referência}

$\mathrm{O}$ agrupamento das amostras possibilitou se conhecer a distribuição das concentrações desses elementos em diferentes níveis, a partir do conhecimento dos teores de silte, argila, CTC, Mn e Fe, ou seja, de acordo com importantes atributos do solo e que estão associadas a processos geoquímicos e estudos pedológicos (Oliveira et al., 1992). Esta distinção pode ser melhor visualizada nos dados encontrados na Tabela 3, em que são mostrados os valores considerados normais em solos brasileiros, tomando-se por base o valor correspondente ao QS (75\%) da distribuição de freqüências dos dados de cada grupo formado.
Tabela 3. Valores considerados normais para o teor de $\mathrm{Cd}, \mathrm{Co}, \mathrm{Cr}$, $\mathrm{Cu}, \mathrm{Ni}, \mathrm{Pb}$ e $\mathrm{Zn}$ em solos não poluídos, proposto para uso como valores de referência $(V R)^{1}$

\begin{tabular}{crrrrrrr}
\hline \multirow{2}{*}{$\begin{array}{c}\text { Grupo } \\
\text { (G) }\end{array}$} & \multicolumn{7}{c}{ Elemento } \\
\cline { 2 - 8 } & $\mathbf{C r}$ & $\mathbf{C o}$ & $\mathbf{N i}$ & $\mathbf{C u}$ & $\mathbf{Z n}$ & $\mathbf{C d}$ & $\mathbf{P b}$ \\
\hline 1 & 55 & 20 & 35 & 119 & 79 & 1,0 & 19 \\
2 & 48 & 10 & 18 & 19 & 44 & 0,8 & 25 \\
3 & 65 & 4 & 25 & 16 & 23 & 1,6 & 16 \\
4 & 35 & 10 & 17 & 12 & 35 & 0,9 & 18 \\
5 & 23 & 4 & 7 & 6 & 12 & 0,4 & 22 \\
6 & 43 & 2 & 12 & 2 & 12 & 0,4 & 3 \\
7 & 19 & 2 & 5 & 3 & 6 & 0,3 & 40 \\
$\mathrm{QSm}^{2}$ & 41 & 8 & 17 & 25 & 30 & 0,8 & 20 \\
\hline
\end{tabular}

${ }^{1}$ Concentração considerada normal para os solos pertencentes a cada grupo e que correspondem ao valor do quartil superior (75\%) da distribuição de frequêencia dos dados amostrais, em cada grupo, Quartil superior médio entre os grupos

Analisando-se os dados da Tabela 3, é possível identificar, no G1, as maiores concentrações para quase todos os metais devido, principalmente, aos maiores teores de Mn e $\mathrm{Fe}$, associados a uma quantidade expressiva de argila, o que, por sua vez, está relacionado com a origem basáltica de vários solos componentes deste grupo. O inverso ocorre no G7 em que a concentração de todos os elementos é inferior à dos demais grupos, exceto para o $\mathrm{Pb}$. Os valores de $\mathrm{Pb}$ para este grupo devem ser vistos com reserva, pois além de não ter sido obtida leitura para $40 \%$ das amostras, a concentração média foi estimada com maior erro. As menores concentrações encontradas neste grupo são, possivelmente, decorrentes do tipo de material de origem associado aos solos deste grupo, predominantemente sedimentar.

Os grupos G6 e G7 são relativamente próximos em termos de concentrações de metais. As variações encontradas são, sem dúvida, devidas aos maiores teores de Fe e Argila no G6 (Tabela 2). Os demais grupos apresentam concentrações intermediárias entre o G1 e G7, havendo tendência geral para que as concentrações de todos os elementos diminuam a partir do G1 (Tabela 3).

Os valores médios do quartil superior (QSm) apresentados na Tabela 3, são próximos dos valores citados por Casarini (2000) como valores de referência de qualidade para metais em solos do estado de São Paulo, cujas concentrações em mg kg-1, são: Cd (0,5), Co (12,5), Cu $(35,1), \operatorname{Cr}(40,2)$, Ni (13,2), Pb (17), Zn (59,9). Esses valores também foram 
calculados com base no QS e apenas o Zn apresenta uma concentração nitidamente superior à que foi obtida para a média do QS, neste estudo. As concentrações correspondentes ao QS podem ser consideradas valores normais para solos com características similares às dos sete grupos (Tabela 2) e são propostas para adoção como VR para concentrações naturais de solos brasileiros.

Além de servir para se obter os VR, os dados de concentração dos metais pesados também foram utilizados para o cálculo do limite máximo, a fim de que a concentração de um elemento fosse admitida como ainda esperada para determinado grupo (Tabela 4); em outras palavras, concentrações superiores a este limite seriam indicativas de que dada amostra não apresenta mais a concentração normal nem o valor máximo, através do qual seria possível se encontrar amostras semelhantes às do grupo em que se enquadra (teores de Silte, Argila, CTC, Mn e Fe). Esses limites foram chamados de limites de tolerância (LT) e correspondem ao valor obtido pela expressão antilog $(m+2 s)$, em que $m$ é média e s o desvio dos dados transformados em $\log _{10}$. Abaixo deste valor estarão contidos cerca de $97,25 \%$ dos casos que podem ocorrer, considerando-se a variação dentro de cada grupo.

Tabela 4. Limite máximo (LT) ${ }^{1}$ proposto para que um nova amostra seja considerada dentro da faixa de concentração esperada para os solos do grupo em que se enquadra

\begin{tabular}{crrrrrrr}
\hline \multirow{2}{*}{$\begin{array}{c}\text { Grupo } \\
\text { (G) }\end{array}$} & \multicolumn{7}{c}{ Elemento } \\
\cline { 2 - 8 } & $\mathbf{C r}$ & $\mathbf{C o}$ & $\mathbf{N i}$ & $\mathbf{C u}$ & $\mathbf{Z n}$ & $\mathbf{C d}$ & $\mathbf{P b}$ \\
\hline 1 & 114 & 44 & 92 & 283 & 149 & 2 & 35 \\
2 & 94 & 34 & 45 & 41 & 92 & 2 & 66 \\
\hline 3 & 131 & 12 & 46 & 48 & 42 & 4 & 31 \\
4 & 81 & 21 & 43 & 41 & 65 & 2 & 60 \\
\hline 5 & 61 & 9 & 21 & 11 & 24 & 1 & 84 \\
6 & 53 & 3 & 19 & 2 & 14 & 1 & 4 \\
7 & 41 & 8 & 10 & 5 & 13 & 1 & 154 \\
\hline
\end{tabular}

${ }^{1} \mathrm{LT}$ (limite de tolerância $)=\operatorname{antilog}_{10}(\mathrm{~m}+2 \mathrm{~s})$, sendo $\mathrm{m}$ a média e $\mathrm{s} 0$ desvio dos dados transformados em $\log _{10}$

Como o objetivo deste trabalho é sugerir valores para caracterização das concentrações de metais em solos, que sejam úteis para interpretações do ponto de vista da poluição ambiental, somente o limite superior foi calculado. A expressão visando aos limites superior e inferior antilog $(\mathrm{m} \pm 2 \mathrm{~s})$ foi utilizada por outros autores para o cálculo de concentrações normais de metais pesados nos solos (Crock et al., 1992; Reaves \& Berrow, 1984; Tobias et al., 1997a)

\section{Avaliação do grau de acerto na classificação das amostras por agrupamento}

Uma maneira de se verificar a qualidade da distribuição das amostras resultante da formação dos grupos, é avaliar o grau de acerto na classificação com a AD. A AD é usada para interpretar grupos de objetos, definidos, a priori, pelos métodos de agrupamento e ordenação (Valentin, 2000). Esta técnica não consiste em formar grupos, já que eles são previamente conhecidos, mas interpretá los com base em variáveis que possam discriminar dois ou mais grupos que ocorram naturalmente (Statsoft, 1999). No presente estudo, como não havia um conhecimento prévio dos grupos que provavelmente se formariam, admitiu-se que os grupos obtidos por semelhança são aqueles que ocorreriam naturalmente, representando ambientes edáficos, comuns no Brasil; como resultado, obteve-se a matriz de classificação para as amostras em cada grupo e a percentagem de acertos em relação à classificação anteriormente obtida na análise de agrupamento (Tabela 5).

Tabela 5. Avaliação do grau de acerto na alocação das amostras nos sete grupos por meio das funções de classificação da análise discriminante

\begin{tabular}{cccc}
\hline $\begin{array}{c}\text { Grupos } \\
\text { (G) }\end{array}$ & Total de amostras & $\begin{array}{c}\text { Casos classificados } \\
\text { corretamente }^{2}\end{array}$ & \% de acerto \\
\hline 1 & 44 & 39 & 89 \\
2 & 32 & 27 & 84 \\
3 & 54 & 52 & 96 \\
4 & 43 & 37 & 83 \\
5 & 29 & 24 & 86 \\
6 & 17 & 17 & 100 \\
\hline 7 & 37 & 33 & 89 \\
\hline Média Geral $^{3}$ & & & 89 \\
\hline
\end{tabular}

${ }^{1}$ Número de amostras classificadas em cada grupo, de acordo com a análise de agrupamento, ${ }^{2}$ Número de casos classificados corretamente em cada grupo, segundo a análise discriminante, ${ }^{3}$ Percentagem média de acertos em cada grupo

Em média, 89\% das amostras foram agrupados em conjuntos que, de fato, são distintos entre si, em função dos teores de silte, argila, CTC, Fe e Mn; entretanto, em média $11 \%$ poderiam estar compondo grupos diferentes daqueles em que foram inicialmente alocados. Embora a percentagem de acertos não tenha sido a ideal, pode-se considerar que a formação dos grupos possui consistência suficiente para a validação dos valores obtidos para a concentração normal dos metais pesados em cada grupo.

Além de possibilitar a avaliação do grau de acerto na distribuição das amostras entre os grupos, a análise discriminante também permite alocar-se novos casos nos grupos já formados. As funções de classificação podem ser usadas para determinar a que grupo uma nova amostra tem maior probabilidade de pertencer e, em geral, os novos casos são classificados no grupo para o qual a função de classificação correspondente resulta no maior valor (Statsoft, 1999). Este procedimento pode ser de grande utilidade na avaliação de áreas suspeitas de estarem poluídas e para o monitoramento de áreas onde se desenvolvem atividades agrícolas ou industriais. Amostras coletadas em áreas cuja concentração natural já não pode ser determinada, podem ser enquadradas em um dos grupos formados (Tabela 2) e, em seguida, pode ser obtido o VR no solo, para cada elemento (Tabela 3), como também o LT (Tabela 4). A comparação direta entre o valor de concentração obtido pela análise da nova amostra e o VR e LT, indicaria se há ou não motivos para se suspeitar que a área em questão contém concentrações do elemento além daquela que normalmente teria, em função das variáveis de solo consideradas para a alocação da amostra no grupo. $\mathrm{Na}$ Tabela 6 são apresentadas as funções de classificação para os sete grupos. 
Tabela 6. Funções de classificação obtidas pela análise discriminante para a alocação das amostras nos grupos previamente formados ${ }^{1}$

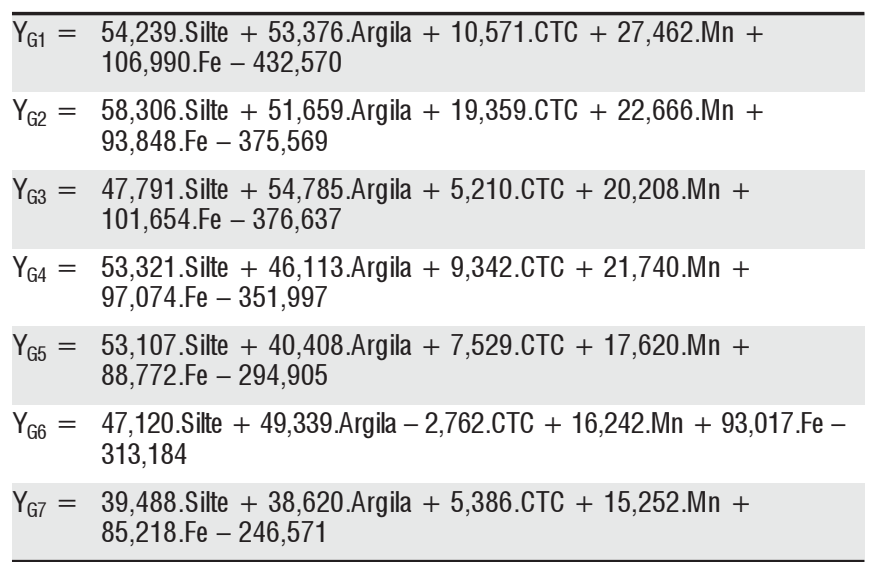

0s coeficientes das funções são para valores das variáveis em $\log _{10}$

\section{Teste para verificar a viabilidade de uso das funções de classificação}

Na tentativa de se verificar se realmente o uso das funções é viável na prática, tomaram-se dez amostras de solo, que não foram utilizadas na formação dos grupos e cujos atributos considerados são mostrados na Tabela 7 .

Tabela 7. Caracterização das amostras usadas para avaliar a eficácia das funções discriminantes na alocação de novos casos*

\begin{tabular}{|c|c|c|c|c|c|c|c|}
\hline Solo & Símbolo & Horizonte & CTC & Silte & Argila & $\mathrm{Fe}$ & $\operatorname{Mn}_{\mathbf{k g}^{-1}}$ \\
\hline \multirow[b]{2}{*}{1} & $\operatorname{LAd}^{1}$ & A & 1,03 & 2.41 & 283 & 4,28 & 236 \\
\hline & LAd & $A B$ & 0,79 & 2,30 & 2,88 & 4,35 & 2,40 \\
\hline \multirow{2}{*}{2} & $\mathrm{LAc}^{2}$ & A1 & 0,87 & 1,90 & 2,46 & 1,00 & 1,42 \\
\hline & LAc & Bw1 & 0,73 & 1,78 & 2,63 & 1,19 & 1,48 \\
\hline \multirow{2}{*}{3} & LAd & A1 & 0,69 & 1,78 & 2,18 & 0,71 & 1,82 \\
\hline & LAd & Bt1 & 0,54 & 1,85 & 2,49 & 1,00 & 1,53 \\
\hline \multirow{2}{*}{4} & $\mathrm{NVd}^{3}$ & A & 0,99 & 2,32 & 2,65 & 4,78 & 2,76 \\
\hline & NVd & Bt & 0,88 & 2,18 & 2,85 & 4,88 & 2,62 \\
\hline \multirow{2}{*}{5} & $\mathrm{CXbe}^{4}$ & A & 1,35 & 2,70 & 2,46 & 4,26 & 2,99 \\
\hline & cXbe & $A B$ & 1,19 & 2,61 & 2,59 & 4,36 & 2,95 \\
\hline
\end{tabular}
Vermelho Distrófico; ${ }^{4}$ Cambissolo Háplico Tb Eutrófico

Os valores para cada função, resultantes da substituição das variáveis (silte, argila, CTC, Mn e Fe) pela concentração de cada uma nas dez amostras, foram comparados entre si para determinar em qual grupo estas deveriam ser alocadas, o que pode ser visto na Tabela 8. O maior valor obtido entre as funções, para cada amostra, indica de qual grupo ela está mais próxima (Statsoft, 1999). As concentrações de metais pesados determinadas por extração com água régia, das dez amostras, foram então comparadas com os VR's e LT's constantes nas Tabelas 3 e 4. Observou-se que a concentração determinada pela análise química foi $100 \%$ dos casos, inferior ao LT e entre 40 e $70 \%$ dos casos o valor determinado foi $£$ VR; os resultados dão uma boa indicação do potencial de uso das funções de classificação para a avaliação do teor de metais em amostras de solo provenientes de áreas sujeitas a atividades antrópicas.
Tabela 8. Classificação de novas amostras nos sete grupos obtidos com uso das funções discriminantes e comparação das concentrações determinadas com aquelas previstas para cada grupo (VR e LT)

\begin{tabular}{|c|c|c|c|c|c|c|c|c|}
\hline \multirow{3}{*}{ Amostra } & \multirow{3}{*}{$\begin{array}{l}\text { Grupo } 1 \\
\text { (G) }\end{array}$} & \multicolumn{7}{|c|}{ Elemento } \\
\hline & & Cd & Co & $\mathrm{Cr}$ & $\mathrm{Cu}$ & $\mathrm{Ni}$ & $\mathrm{Pb}$ & $\mathrm{Zn}$ \\
\hline & & \multicolumn{7}{|c|}{$\mathrm{mg} \mathrm{kg}^{-1}$} \\
\hline LAd & 2 & 1,3 & 3,9 & 28 & 9 & 13 & 5 & 30 \\
\hline LAd & 3 & 2,3 & 3,6 & 54 & 9 & 20 & nd & 30 \\
\hline LAc & 7 & 0,4 & 2,0 & 21 & $\mathrm{nd}^{4}$ & 7 & nd & 8 \\
\hline LAc & 7 & 0,6 & 2,8 & 35 & 2 & 10 & nd & 11 \\
\hline LAd & 7 & 0,2 & 2,3 & 12 & $<0,5$ & 3 & $<0,5$ & 4 \\
\hline LAd & 7 & 0,3 & 2,7 & 24 & nd & 6 & nd & 6 \\
\hline NVd & 1 & 1,8 & 26,5 & 27 & 61 & 26 & 15 & 47 \\
\hline NVd & 1 & 2,5 & 24,7 & 34 & 59 & 28 & 17 & 60 \\
\hline cXbe & 2 & 1,2 & 12,3 & 17 & 25 & 16 & 21 & 82 \\
\hline CXbe & 2 & 1,1 & 14,5 & 22 & 34 & 17 & 16 & 74 \\
\hline$\leq \mathrm{VR}^{2}$ & $\%$ & 40 & 40 & 70 & 50 & 70 & 100 & 60 \\
\hline$<\mathrm{LT}^{3}$ & $\%$ & 100 & 100 & 100 & 100 & 100 & 100 & 100 \\
\hline
\end{tabular}

Grupo do qual a amostra mais se aproxima. ${ }^{2}$ Percentagem de amostras cuja concentração foi inferior ou igual ao VR do grupo em que se enquadra. ${ }^{3}$ Percentagem de amostras cuja concentração foi inferior ao LT do grupo em que se enquadra. ${ }^{4}$ Não detectado

\section{CONCLUSÕES}

1. As concentrações de Cd, Co, Cr, Cu, Ni, Pb e Zn definidas em cada grupo poderiam ser utilizadas para a avaliação preliminar de áreas com suspeita de poluição ou com uso agrícola, cujos solos possuam características similares às deste estudo.

2. As funções de classificação mostraram ser uma ferramenta adequada para a alocação de novas amostras, nos grupos previamente estabelecidos.

\section{LITERATURA CITADA}

Alloway, B. J. The origin of heavy metals in soils. In: Alloway, B. J. (ed.) Heavy metals in soils. 2.ed. Glasgow: Blackie Academic \& Professional, 1995. p.38 57.

Amaral Sobrinho, N. M. B. Interação dos metais pesados de resíduos siderúrgicos com um podzólico Vermelho Amarelo, 1993. Viçosa UFV. 163p. Tese Doutorado

Broschat, T. K. Principal component analysis in horticultural research. Hortscience, Alexandria, v.14, n.2, p.114 117, 1979.

Casarini, D. C. P. Proposta de valores de referência de qualidade e intervenção para solos e águas subterrâneas no estado de São Paulo. In: Seminário Internacional sobre Qualidade de Solos e Águas Subterrâneas, 2., 2000, São Paulo. Anais. São Paulo: CETESB, 2000. 165p.

Correia, J. R. Uso de técnicas multivariadas no estudo das inter relações de características do solo e a produtividade do eucalipto.. Viçosa, UFV, 1993, 110p. Dissertação Mestrado

Crock, J. G.; Severson, R. C.; Gough, L. P. Determining baselines and variability of elements in plants and soils near the Kenai National Wildlife Refuge, Water, Air, and Soil Pollution, Netherlands, v.63, n.3/4, p.253-271, 1992.

Cruz, C. D.; Regazzi, A. J. Modelos biométricos aplicados ao melhoramento genético. Viçosa: UFV, 2001. 390p. 
EMBRAPA - Empresa Brasileira de Pesquisa Agropecuária.Centro Nacional de Pesquisa de Solos. Sistema brasileiro de classificação de solos. Brasília: Embrapa Produção de Informação, 1999. 412p.

Fadigas, F. S. Estimativa das concentrações naturais (pseudo total) de $\mathrm{Cd}, \mathrm{Co}, \mathrm{Cr}, \mathrm{Cu}, \mathrm{Ni}, \mathrm{Pb}$ e $\mathrm{Zn}$ em solos brasileiros e proposição de valores de referência utilizando técnicas da estatística multivariada. Seropédica. UFRRJ, 2002. 116p. Tese Doutorado

Fadigas, F. S.; Amaral Sobrinho, N. M. B.; Mazur, N.; Anjos, L. H. C.; Freixo, A. A. Concentrações naturais de metais pesados em algumas classes de solos brasileiros. Bragantia, Campinas, v.2, p.151-159, 2002.

Furlani, P. R.; Bataglia, O. A.; Valadares, J. M. A. Cobalto em solos do Estado de São Paulo. Revista Brasileira de Ciência do Solo, Piracicaba, v.1, p.65-67, 1977.

Klamt, E.; van Reeuwijk, L. P. Evaluation of morphological, physical and chemical characteristics of ferralsols and related soils. Revista Brasileira de Ciência do Solo, Viçosa, v.24, n.3, p.573 587, 2000.

Lavado, R. S.; Rodriguez, M. B.;Sheiner, I. D.; Taboada, M. A.; Rubio, G.; Alvarez. R.; Alconada, M.; Zubillaga, M. S. Heavy metals in soils of Argentina: comparison between urban and agricultural soils. Communications in Soil Science and Plant Analysis, Athens, v.29, n.11 14, p.1913-1917, 1998.

Lemos, M. M. G. Metodologia adotada para o estabelecimento dos valores de referência de qualidade para solos e águas subterrâneas no estado de São Paulo. In: CETESB. Prevenção e controle da poluição do solo e das águas subterrâneas. São Paulo: CETESB, 2000. p.68-77.

Lima, P. C. Micromorfolgia de horizontes B de Latossolos do Sudeste e Sul do Brasil. In: EMBRAPA - Empresa Brasileira de Pesquisa Agropecuária. Investigações coligadas em Latossolos do Sudeste e Sul do Brasil. Rio de Janeiro: EMBRAPASNCLS, 1984. p.168-188.

Mazur, N. Níquel, chumbo, zinco e cobre em solos que receberam composto de resíduos sólidos urbanos. Viçosa, UFV,1997 135p. Tese Doutorado

Moura, C. V. A. Aplicação de tratamento estatístico multivariante em dados geoquímicos de solo no mapeamento geológico na província de Carajás (alvo 2 - corpo 4). Revista Brasileira de Geociências, São Paulo, v.15, n.3, p.241 248, 1985.

Oliveira, J. B.; Jacomine, P. K. T.; Camargo, M. N. Classes gerais de solos do Brasil. 2.ed. Jaboticabal: FUNEP, 1992. 201p.

Oliveira, T. S. Metais pesados como indicadores de materiais de origem de solos.. Viçosa, UFV, 1996, 128p. Tese Doutorado

Oliveira, T. S.; Costa, L. M.; Cruz, C. D. Importância relativa dos metais pesados do solo na identificação e separação de materiais de origem. Revista Ceres, Viçosa, v.45, n.260, p.359 371, 1998.

Parks, J. M. Cluster analysis applied to multivariate geologic problems. Journal of Geology, Chicago, v.74, n.5, p.703 715, 1966.
Pérez, D. V.; Saldanha, M. F. C.; Meneguelli, N. A.; Moreira, J. C; Vaitsman, D. S. Geoquímica de alguns solos brasileiros. Pesquisa em andamento. Rio de Janeiro, n.4, p.114, 1997.

Qian, J.; Wang, Z.; Shan, X.; Tu Q.; Wen B.; Chen, B. Evaluation of plant availability of soil trace metals by chemical fractionation and multiple regression analysis. Environmental Pollution, Amsterdam, v.91, n.3, p.309 315, 1996.

Ramalho, J. F. P. G.; Amaral Sobrinho, N. M. B.; Velloso, A. C. $X$. Acúmulo de metais pesados em solos cultivados com canade-açucar pelo uso contínuo de adubação fosfatada e água de irrigação. Revista Brasileira de Ciência do Solo, Viçosa, v.23, n.4, p.971 979, 1999.

Ramalho, J. F. P. G.; Amaral Sobrinho, N. M. B.; Velloso, A. C. $\mathrm{X}$. Contaminação da microbacia de Caetés com metais pesados pelo uso de agroquímicos. Pesquisa Agropecuária Brasileira, Brasília, v.35, p.1289 1303, 2000.

Reaves, G. A.; Berrow, M. L. Total lead concentration in Scottish soils. Geoderma, Amsterdam, v.32, p.1 8, 1984.

Ross, S. M. Toxic metals in soil-plant systems. Chichester: John Willey \& Sons, 1994. 469p.

Rovers, H.; Camargo, O. A.; Valadares, J. M. A. S. Níquel total e solúvel em DTPA em solos do Estado de São Paulo. Revista Brasileira de Ciência do Solo, Piracicaba, v.7, n.3, p.217 220, 1983.

Santos Filho, A.; Rocha, H. O. O cobre em solos do Estado do Paraná. Revista do Setor de Ciências Agrárias, Curitiba, v.4, n.1 2, p.23-26, 1982.

Souza, M. L. P.; Andreoli, C. V.; Amaral, M. B., et al. Levantamento preliminar dos teores de metais pesados em alguns solos do Paraná. Revista Sanare, Curitiba, v.5, n.5, p.68-75, 1996.

Statsoft, INC. Statistica for Windows - computer program manual. Tulsa (OK): StatSoft, 1999.

Tobias, F. J.; Bech, J.; Algarra, P. S. Establishment of the background levels of some trace elements in soils of NE Spain with probability plots. The Science of Total Environment, Amsterdam,v.206, p.255 265, 1997a.

Tobias, F. J.; Bech, J.; Algarra, P. S. Statistical approach to discriminate background and anthropogenic input of trace elements in soils of Catalonia, Spain. Water, Air, and Soil Pollution, Netherlands, v.100, p.63-78, 1997b.

Valadares, J. M. A. S. Cobre em solos do Estado de São Paulo: cobre total. Bragantia, Campinas, v.34, n.4, p.125-132, 1975.

Valadares, J. M. A. S.; Catani, R. A. Zinco em solos do Estado de São Paulo: Zinco total. Bragantia, Campinas, v.34, p.134 139, 1975.

Valentin, J. L. Ecologia numérica - uma introdução à análise multivariada de dados ecológicos. Rio de Janeiro: Interciência, 2000. 117p.

Wang, H. K. Research methodology for determining background levels of trace elements in Chinese soils. Environmental Geochemistry and Health, 1(Special Issue), Amsterdam, p.397-413, 1994. 